\title{
TMEM165, a Golgi transmembrane protein, is a novel marker for hepatocellular carcinoma and its depletion impairs invasion activity
}

\author{
JEE-SAN LEE $^{1 *}$, MI-YEUN KIM ${ }^{1,8^{*}}$, EUN-RAN PARK $^{1}$, YAN NAN SHEN $^{1,10}$, JU-YEON JEON $^{1}$, \\ EUNG-HO CHO ${ }^{2}$, SUN-HOO PARK ${ }^{3}, \mathrm{CHUL} \mathrm{JU} \mathrm{HAN}^{4}$, DONG WOOK $\mathrm{CHOI}^{5}$, JA JUNE JANG ${ }^{6}$, \\ KYUNG-SUK SUH ${ }^{7}$, JUNGIL HONG ${ }^{8}$, SANG BUM KIM $^{2}$ and KEE-HO LEE ${ }^{1,9}$ \\ ${ }^{1}$ Division of Radiation Cancer Research, Departments of ${ }^{2}$ Surgery, ${ }^{3}$ Pathology and ${ }^{4}$ Internal Medicine, \\ Division of Radiological and Clinical Research, Korea Institute of Radiological and Medical Sciences, Seoul 01812; \\ ${ }^{5}$ Department of Surgery, Samsung Medical Center, Sungkyunkwan University School of Medicine, Seoul 06351; \\ ${ }^{6}$ Departments of Pathology and ${ }^{7}$ Surgery, Seoul National University School of Medicine, Seoul 08226; \\ ${ }^{8}$ Division of Applied Food System and ${ }^{9}$ Department of Biotechnology, College of Natural Science, \\ Seoul Women's University, Seoul 01797, Republic of Korea; ${ }^{10}$ Key Laboratory of Radiobiology, \\ School of Public health, Jilin University, Changchun, Jilin 130012, P.R. China
}

Received November 8, 2017; Accepted June 26, 2018

DOI: $10.3892 /$ or.2018.6565

\begin{abstract}
Transmembrane protein 165 (TMEM165), a Golgi protein, functions in ion homeostasis and vesicular trafficking in the Golgi apparatus. While mutations in TMEM165 are known to cause human 'congenital disorders of glycosylation', a recessive autosomal metabolic disease, the potential association of this protein with human cancer development has not been explored to date. In the present study, we revealed that TMEM165 is overexpressed in HCC and its depletion weakens the invasive activity of cancer cells through suppression of matrix metalloproteinase-2 (MMP-2) expression. Levels of TMEM165 mRNA and protein were clearly increased in HCC patient tissues and cell cultures. Quantitative real-time RT-PCR analysis of fresh HCC tissues $(n=88)$ revealed association of
\end{abstract}

Correspondence to: Dr Kee-Ho Lee, Division of Radiation Cancer Research, Korea Institute of Radiological and Medical Sciences, 75 Nowon-Ro, Nowon-Ku, Seoul 01812, Republic of Korea

E-mail:khlee@kirams.re.kr

Dr Sang Bum Kim, Department of Surgery, Korea Institute of Radiological and Medical Sciences, 75 Nowon-Ro, Nowon-Ku, Seoul 01812, Republic of Korea

E-mail: lull2@kirmas.re.kr

*Contributed equally

Abbreviations: HCC, human hepatocellular carcinoma; AFP, $\alpha$-fetoprotein; AST, aspartate aminotransferase; ALT, alanine transaminase; MMP, matrix metalloproteinase

Key words: TMEM165, Golgi, hepatocellular carcinoma, invasion, MMP-2
TMEM165 overexpression with more frequent macroscopic vascular invasion, microscopic serosal invasion and higher $\alpha$-fetoprotein levels. Notably, depletion of TMEM165 led to a marked decrease in the invasive activity of two different HCC cell types, Huh7 and SNU475, accompanied by downregulation of MMP-2. Our collective findings clearly indicated that TMEM165 contributed to the progression of HCC by promoting invasive activity, supporting its utility as a novel biomarker and therapeutic target for cancer.

\section{Introduction}

Hepatocellular carcinoma (HCC) is the most common form of primary liver cancer in adults and the third leading cause of cancer-related mortality worldwide (1). HCC occurs most frequently in patients with chronic liver disease and cirrhosis (2). The common risk factors of HCC include hepatitis $\mathrm{B}$ and $\mathrm{C}$ virus infection, heavy consumption of alcohol, exposure to aflatoxin B1 and non-alcoholic steatohepatitis (3). Despite significant advances in diagnosis and treatment, liver resection and transplantation are considered the only curative options at present (3). To date, no effective treatment strategies have been developed for patients with advanced HCC, including the approved therapeutic agent, sorafenib (4), owing to chemoresistance and excessive cytotoxicity resulting in dismal prognosis (5). Therefore, identification of early and effective diagnostic markers and therapeutic targets for this disease remains an urgent unmet medical need.

The discovery of genes involved in tumor progression has prompted research into various methods of therapeutic intervention. Based on advances in genome-wide technology in cancer research, we established a microarray dataset to identify the genes responsible for dismal prognosis in earlier studies (6-8). In the present study, transmembrane protein 165 
(TMEM165), a Golgi protein (9), was shown to be associated with aggressive characteristics of HCC. TMEM165 is a highly conserved hydrophobic protein of 324 amino acids that contains 7 transmembrane-spanning domains according to topology predictions (10). Recent research has revealed that mutations in TMEM165 were associated with a rare autosomal recessive disease designated 'congenital disorders of glycosylation' leading to defects in metabolic processes through impairment of galactosylation and sialylation of $\mathrm{N}$-glycoproteins (10-14). The majority of congenital disorders of glycosylation are caused by defects in glycosylation machinery components, such as SLC35A1, B4GALT1 and MGAT2 (15-17). In contrast to the glycosylation-governing proteins, TMEM165 maintains Golgi ion homeostasis and vesicular Golgi trafficking (18). While TMEM165 has been extensively characterized in association with congenital disorders of glycosylation, its relationship with cancer remains to be determined. HCC-related proteins have been identified in multiple cellular compartments, including the nucleus, cytoplasm, mitochondria and plasma membrane. However, association of Golgi proteins with $\mathrm{HCC}$ has rarely been documented in the literature to date. To the best of our knowledge, GP73 (a 73-kDa Golgi transmembrane protein) is the protein that has been relatively well documented as a useful serum biomarker for HCC and shown to be involved in the progression of benign liver disease $(17,19,20)$. In the present study, we identified a Golgi transmembrane protein, TMEM165, that is overexpressed in HCC patient tissues. TMEM165 expression was significantly associated with high levels of $\alpha$-fetoprotein as well as presence of macroscopic vascular and serosal invasion. Consistently, TMEM165 depletion attenuated invasion activities of HCC cells via decrease in MMP-2 expression. Our findings revealed that TMEM165 could serve as a novel HCC marker associated with cancer aggressiveness and presents a potential therapeutic target.

\section{Materials and methods}

Patients and tissue samples. HCC tissues were acquired from patients with HCC subjected to hepatic surgery between September 1992 and December 2004 at Korea Cancer Center Hospital. A total of $88 \mathrm{HCCs}$ used in the present study included 33 pair-matched HCC and adjacent liver tissues, and 55 HCC tissues. Normal liver tissues were obtained from patients with colon or rectal cancer, metastasized into liver, who underwent surgical resection at Seoul National University Hospital and Korea Cancer Center Hospital. The adjacent liver tissues around these metastatic cancers were regarded and used as a normal liver $(\mathrm{n}=15)$ when liver fibrosis as well as HCC were not observed in the patients. Our study was approved by the Institutional Review Boards of Korea Cancer Center Hospital and Seoul National University Hospital. Written informed consent was either waived by the Institutional Review Board of Korea Cancer Center Hospital or received from patients at Seoul National University Hospital, respectively. Clinicopathological information collected retrospectively was utilized to examine associations between TMEM165 expression patterns and HCC characteristics. Fibrosis was graded on a scale of 0-4 (stage 0 , not present; stage 1, portal; stage 2, periportal; stage 3, septal; and stage 4, cirrhosis).
Cell culture and siRNA transfection. Cell lines were obtained from two institutions. The cell lines, SNU354, SNU398, SNU449, SNU475, SNU739 and Huh7 were purchased from the Korean Cell Line Bank (Seoul, South Korea) and the cell lines, Hep3B, HepG2, BJ, IMR90, WI38, normal Small Airway (PCS-301-010) and Prostate (PCS-440-010) epithelial cells from the American Type Culture Collection (ATCC; Manassas, VA, USA). Huh7, SNU354, SNU398, SNU449, SNU475 and SNU739 cells were cultured in RPMI-1640 medium (LM 011-01; Welgene, Daegu, Korea) and BJ, IMR90, WI38, HepG2 and Hep3B were cultured in MEM (LM 007-07; Welgene) supplemented with $10 \%$ (w/v) fetal bovine serum (FBS; JR Scientific, Inc., Woodland, CA, USA) and $1 \%(\mathrm{w} / \mathrm{v})$ penicillin/streptomycin (Welgene) at $37^{\circ} \mathrm{C}$. Normal small Airway and Prostate epithelial cells were cultured in Airway Epithelial Basal media (ATCC PCS 300-030) supplemented with Bronchial Epithelial Cell Growth kit (ATCC PCS-300-040) and Prostate Epithelial Cell Basal Medium (ATCC PCS-440-030) supplemented with Prostate Epithelial Cell Growth kit (ATCC PCS-440-040), respectively. For knockdown of TMEM165, siRNA transfection was performed using Lipofectamine RNA iMAX reagent (cat. no. 13778030; Invitrogen; Thermo Fisher Scientific, Inc., Waltham, MA, USA) according to the manufacturer's instructions. The following siRNA sequences were used: TMEM165 siRNA\#1, 5'-AGCCAUCAUGGCAAUGCGCUAUU-3' (sense) and 5'-UAGCGCAUUGCCAUGAUGGCUUU-3' (antisense); and TMEM165 siRNA\#2, 5'-UUGGGUAGGACACCCAAUAUA UU-3' (sense) and 5'-UAUAUUGGGUGUCCUACCCAA UU-3' (antisense).

RNA extraction and cDNA synthesis. Total RNA was extracted from cultured cells and fresh tissues using the RNeasy Mini Kit (cat. no. 74106; Qiagen, Inc., Valencia, CA, USA) according to the manufacturer's protocol. The quality and concentration of isolated total RNA were determined using a NanoDrop ND-1000 spectrophotometer (NanoDrop; Thermo Fisher Scientific, Inc., Wilmington, DE, USA). RNA samples were stored at $-80^{\circ} \mathrm{C}$ until gene expression analysis. cDNA was synthesized using the iScript reverse transcriptase kit (cat. no. 170890; Bio-Rad Laboratories, Inc., Hercules, CA, USA) in keeping with the manufacturer's protocol.

Quantitative and semi-quantitative RT-PCR. Quantitative RT-PCR was performed with the KAPA SYBR FAST Universal qPCR kit (kit code: KK4301; Kapa Biosystems, Inc., Boston, MA, USA) on the CFX96 Real-Time RT-PCR Detection System (Bio-Rad Laboratories, Inc.). The primer sequences used for real-time RT-PCR were: TMEM165 forward, 5'-GGCAGTAATTGGAGGAAGAATGATAGC-3' and reverse, 5'-ACCAGAATCAGGGCTTATAAATAGT GC-3'; and $\beta 2$-microglobulin forward, 5'-AAGGACTGGTCT TTCTATCTCTTGTA-3' and reverse, 5'-ACTATCTTGG GGTGTGACAAAGTC-3'. Median Ct values from triplicate experiments were used for statistical analyses and TMEM165 levels were normalized to median $\beta 2$-microglobulin expression. Relative TMEM165 expression in HCC and adjacent liver tissues, compared to that in normal liver tissues, was analyzed using the comparative threshold cycle $2^{-\Delta \Delta \mathrm{Ct}}$ method. Semi-quantitative RT-PCR was performed using 
the Maxime PCR PreMix kit (cat. no. 25185; iNtRON Biotechnology, Kyungi-do, Korea) and the following primers: TMEM165 forward, 5'-GGCAGTAATTGGAGG AAGAATGATAGC-3' and reverse, 5'-ACCAGAATCAGG GCTTATAAATAGTGC-3'; $\beta 2$-microglobulin forward, 5'-GTGCTCGCGCTACTCTCTCT-3' and reverse, 5'-CGG CAGGCATACTCATCTTT-3'; MMP-1 forward, 5'-ACA AAGTTGATGCAGTTTTCA-3' and reverse, 5'-GAATCC ATAAGCCACAAACTT-3'; MMP-2 forward, 5'-ATCTTT GCTGGAGACAAATTC-3' and reverse, 5'-AACTTCACG CTCTTCAGACTT-3'; MMP-9 forward, 5'-AACTTTGAC AGCGACAAGAA-3' and reverse, 5'-TTGAACAAATAC AGCTGGTTC-3'; MMP-13 forward, 5'-CCCAAAATTTTC TACCTCTGA-3' and reverse, 5'-TTTTGATGATGATGA AACCTG-3'; $\beta$-actin forward, 5'-GGACTTCGAGCAAGA GATGG-3' and reverse, 5'-AGCACTGTGTTGGCGTAC AG-3'; RPS26 forward, 5'-CCAAGGACAAGGCCATTA AG-3' and reverse, 5'-AGCACCCGCAGGTCTAAATC-3'; and GAPDH forward, 5'-GTCAGTGGTGGACCTGAC CT-3' and reverse, 5'-TGCTGTAGCCAAATTCGTTG-3'. The optimal PCR cycle for each primer set was determined by monitoring PCR products harvested at 2-cycle intervals with at least 3 different cycles. PCR amplification was performed with an initial 2 min of denaturation at $94^{\circ} \mathrm{C}$, followed by cycling a $20 \mathrm{sec}$ denaturation at $94^{\circ} \mathrm{C}, 10 \mathrm{sec}$ annealing at $55^{\circ} \mathrm{C}$ and $30 \mathrm{sec}$ extension at $72^{\circ} \mathrm{C}$ with a final 5 min extension at $72^{\circ} \mathrm{C}$. The final PCR products were loaded on agarose gels and mRNA levels quantitated using NIH ImageJ software (http://rsb.info.nih.gov/ij).

Western blot analysis. Total tissue samples were homogenized in lysis buffer [50 mM Tris- $\mathrm{HCl}, \mathrm{pH} 7.4,150 \mathrm{mM}$ $\mathrm{NaCl}, 5 \mathrm{mM}$ EDTA, $1 \%(\mathrm{v} / \mathrm{v})$ Nonidet P-40, 0.1\% (w/v) sodium dodecyl sulfate, $0.5 \%(\mathrm{w} / \mathrm{v})$ sodium deoxycholate] including protease inhibitor cocktail (cat. no. P3100-010; GenDEPOT, Inc., Barker, TX, USA) and placed on ice for $20 \mathrm{~min}$. After incubation, tissues were centrifuged for $20 \mathrm{~min}$ at $13,000 \mathrm{x} \mathrm{g}$ at $4^{\circ} \mathrm{C}$ and the supernatant fractions were collected. Quantified and sampled proteins were separated on a $12.0 \%(\mathrm{w} / \mathrm{v})$ SDS gel and transferred to nitrocellulose membranes (cat. no. 10401396; Whatman, Maidstone, UK). Next, the membranes were blocked with $5 \%$ (w/v) skim milk in TBS with Tween-20 (TBS-T) buffer $25 \mathrm{mM}$ Tris- $\mathrm{HCl}$, $\mathrm{pH}$ 7.4, $140 \mathrm{mM} \mathrm{NaCl}, 2.7 \mathrm{mM} \mathrm{KCl}, 0.05 \%$ (w/v) Tween-20] for $1 \mathrm{~h}$ at room temperature, followed by incubation with primary antibodies against TMEM165 (diluted to 1:1,000; cat. no. 20485-1-AP; ProteinTech Group, Rosemont, IL, USA) and GAPDH (diluted to 1:2,000; cat. no. sc-25778; Santa Cruz Biotechnology, Inc., Santa Cruz, CA, USA) for $1 \mathrm{~h}$ at room temperature. After washing 3 times with TBS-T, the membranes were treated with horseradish peroxidase-conjugated secondary antibody (diluted to 1:3,000; cat. nos. A120-101P and A90-116P; Bethyl Laboratories, Inc., Montgomery, TX, USA) at room temperature for $1 \mathrm{~h}$. Following 3 further washes with TBS-T, the membranes were reacted with Luminol chemiluminescent reagent (cat. no. sc-2048; Santa Cruz Biotechnology, Inc.) according to the manufacturer's protocol. The intensities of the band were analyzed using the NIH ImageJ software (National Institutes of Health, Bethesda, MD, USA).
A

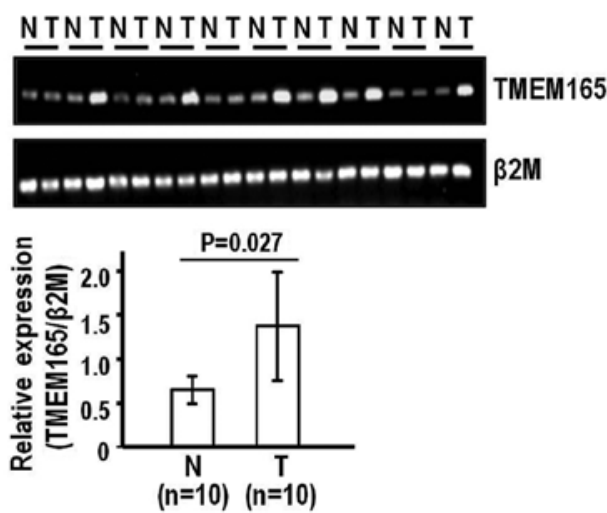

B
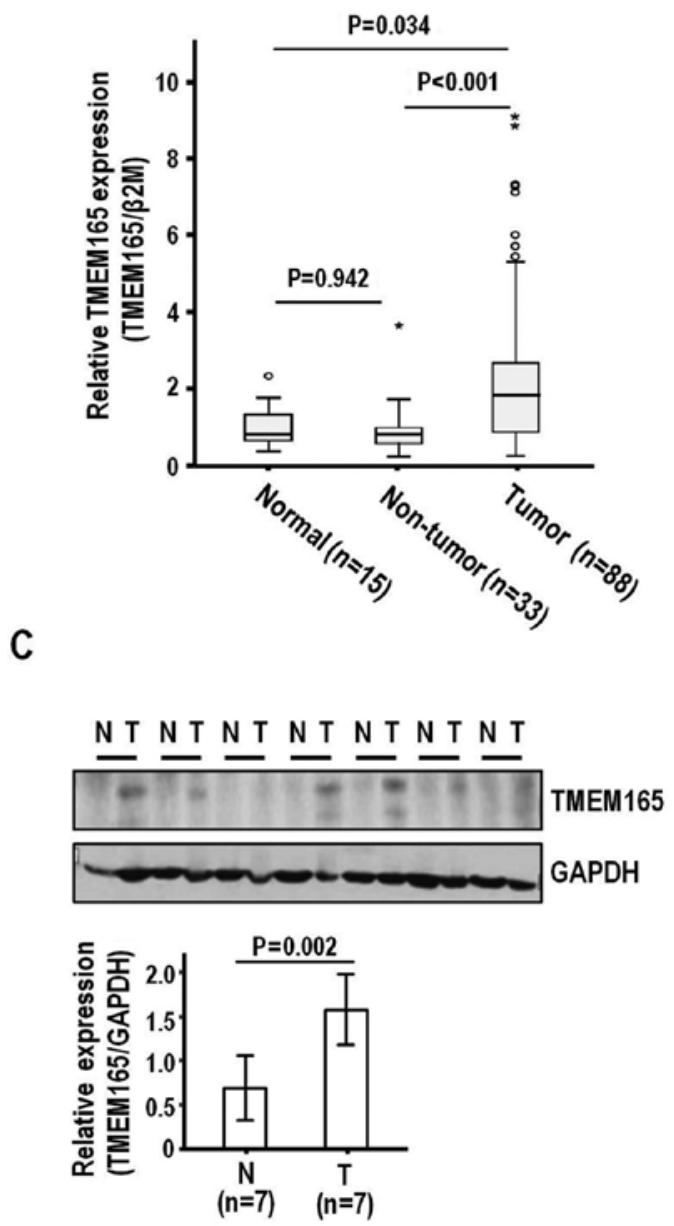

Figure 1. TMEM165 is overexpressed in HCC. (A) TMEM165 mRNA was visualized via semi-quantitative RT-PCR in 10 pair-matched HCC (T) and adjacent liver tissues (N). (B) Real-time RT-PCR results revealing relative mRNA levels of TMEM165 quantitated in normal $(\mathrm{n}=15)$, adjacent liver $(\mathrm{n}=33)$, and HCC tissues $(\mathrm{n}=88)$. The sample set analyzed included 33 pair-matched tissues as indicated in Materials and methods. P-values were calculated by ANOVA Tukey's post hoc test. The horizontal line in the box plot is the median value, and the symbols, open circle $\left({ }^{\circ}\right)$ and asterisk $\left(^{*}\right)$ on the bars indicate outlier and extreme outlier samples, respectively, with $95 \%$ confidence interval. (C) Protein expression of TMEM165 in 7 pair-matched $\mathrm{HCC}$ and adjacent liver tissues determined using western blotting. $\beta 2-$ Microglobulin $(\beta 2 \mathrm{M})$ and GAPDH were used as internal controls. The tissues used for $\mathrm{A}$ and $\mathrm{C}$ were randomly selected from the 33 pair-matched set. All semi-quantitative RT-PCR and western blotting experiments were repeated at least 3 times. The densitometry analysis was performed using ImageJ software. P-values were calculated by a two-sample t-test. TMEM165, transmembrane protein 165; HCC, human hepatocellular carcinoma. 
Table I. Patient demographics and pathological data $(n=88)$.

\begin{tabular}{lll}
\hline Variables & \multicolumn{1}{c}{ Classification } & Distribution \\
\hline Sex & Male : Female & $77: 11$ \\
Age (years) & Year, mean \pm SD (range) & $52.05 \pm 10.79(25-74)$ \\
Etiology & Hepatitis B : Hepatitis C & $85: 03$ \\
Total bilirubin & $<1 \mathrm{mg} / \mathrm{dl}: \geq 1 \mathrm{mg} / \mathrm{dl}$ & $59: 29$ \\
AFP & $<20 \mathrm{ng} / \mathrm{dl}: \geq 20 \mathrm{ng} / \mathrm{dl}$ & $38: 49$ \\
& $<50 \mathrm{ng} / \mathrm{dl}: \geq 50 \mathrm{ng} / \mathrm{dl}$ & $47: 40$ \\
& $<200 \mathrm{ng} / \mathrm{dl}: \geq 200 \mathrm{ng} / \mathrm{dl}$ & $55: 32$ \\
AST & IU/l, mean \pm SD (range) & $51.92 \pm 31.07(15-182)$ \\
ALT & IU/l, mean \pm SD (range) & $46.25 \pm 25.21(10-130)$ \\
Child-Pugh classification & A : B and C & $70: 07$ \\
Tumor size & $\mathrm{cm}$ mean \pm SD (range) & $5.8 \pm 3.25(1.0-24.0)$ \\
Tumor number & Single : Multiple & $59: 17$ \\
Macroscopic vascular invasion & No : Yes & $78: 5$ \\
Microscopic vascular invasion & No : Yes & $34: 39$ \\
Serosal invasion & No : Yes & $68: 12$ \\
Edmondson grade & I : II : III : VI & $11: 48: 27: 1$ \\
Fibrosis & $0: 1: 2: 3: 4$ & $3: 7: 16: 24: 29$
\end{tabular}

${ }^{a}$ Edmondson-Steiner histological grade. In our present patient cohort, the clinicopathological information collected retrospectively has missing data, since some parameters were not obtained from all patients.AFP, $\alpha$-fetoprotein;AST, aspartate aminotransferase; ALT, alanine transaminase .

Clonal survival analysis. Huh7 and SNU475 cells were transiently transfected with control and TMEM165 siRNAs. At $24 \mathrm{~h}$ after transfection, 1,000 cells were seeded on a 6-well plate. Cells were fixed with $10 \%(\mathrm{w} / \mathrm{v})$ formaldehyde and stained with $0.1 \%(\mathrm{w} / \mathrm{v})$ crystal violet 10 days after seeding. Colonies were counted using digital images obtained with ImageJ. All experiments were performed in triplicate.

Invasion analysis. Polycarbonate membrane Transwell inserts (cat. no. 354480; Corning Inc., Corning, NY, USA) were coated with $20 \mu \mathrm{g} / \mathrm{ml}$ Matrigel. Following transient transfection, Huh7 and SNU475 cells $\left(2 \times 10^{4}\right)$ were seeded on the upper compartment of the chamber in serum-free medium. As a chemoattractant, complete medium containing $10 \%$ (v/v) FBS was placed in the bottom compartment. After 24 and $48 \mathrm{~h}$ of incubation, the cells were fixed, stained with Hemacolor ${ }^{\circledR}$ solution (Merck KGaA, Darmstadt, Germany) and counted under a light microscope in 4 different fields. All experiments were performed in triplicate.

Statistical analysis. Statistical analysis was performed using SPSS v.23.0 software (IBM Corp., Armonk, NY, USA). Receiver operating characteristic (ROC) curves were generated to assess the optimal cut-off point for TMEM165 expression. A one-way analysis of variance (ANOVA) with post hoc Tukey's HSD test was conducted to compare the expression levels of TMEM165 in normal, non-tumor and tumor samples. Correlations between TMEM165 expression and clinicopathological parameters were determined using a 2-sample t-test, Pearson's correlation test, Chi-squared $\left(\chi^{2}\right)$ and Fisher's exact tests. For the comparison of invasion and clonal survival, ANOVA with post hoc Dunnett's test was performed. The data were considered significant at $\mathrm{P}<0.05$.

\section{Results}

TMEM165 is overexpressed in HCC. To establish whether TMEM165 is involved in the pathogenesis of HCC, we examined its expression patterns in tumor and adjacent liver tissue specimens obtained from HCC patients subjected to surgical resection. Semi-quantitative RT-PCR disclosed higher TMEM165 expression in HCC than adjacent liver tissues ( $\mathrm{P}=0.027$ ) (Fig. 1A). To confirm TMEM165 expression, we performed quantitative real-time RT-PCR on an extended HCC sample set $(n=88)$ (Table I) comprised of background liver cirrhosis $(n=29)$ and fibrosis $(n=3: 7: 16: 24: 29$ for grades $0: 1: 2: 3: 4$, respectively). Similar to semi-quantitative RT-PCR findings, real-time RT-PCR revealed significantly elevated TMEM165 expression in HCC, compared to adjacent non-tumor liver $(\mathrm{P}<0.001)$ and normal liver tissues $(\mathrm{P}=0.034)$ (Fig. 1B). In contrast to tumor tissues, adjacent liver tissues did not exhibit a significant difference in TMEM165 expression, compared to normal liver tissues $(\mathrm{P}=0.942)$. When expression levels were adjusted and compared with those of normal liver tissues, the mean increases in TMEM165 expression in HCC and adjacent liver tissues were 2.40- and 1.02-fold (median, 1.89- and 0.99-fold, respectively). Consistent with the mRNA expression data, the protein levels were higher in HCC than adjacent liver tissues $(\mathrm{P}=0.002)$ (Fig. 1C). The collective findings clearly demonstrated TMEM165 overexpression in HCC. 
TMEM165 overexpression is clinically associated with macroscopic vascular invasion, microscopic serosal invasion, and high $\alpha$-fetoprotein levels of HCC. Next, we examined the potential association of TMEM165 mRNA levels with clinicopathological parameters to ascertain whether overexpression has a clinical impact on HCC. Variations in TMEM165 expression determined based on real-time analysis were mostly $<2$.0-fold between normal and adjacent liver tissues. To further verify an optimal cut-off point for TMEM165 expression, we performed ROC analysis using SPSS software. In the analysis, the clinical parameters, AFP level ( $<50 \mathrm{vs} . \geq 50 \mathrm{ng} / \mathrm{ml}$ ), macroscopic invasion (no and yes) and serosal invasion (no and yes) were associated with TMEM165 $(\mathrm{P}<0.05)$. The optimal cut-off points of TMEM165 expression for these parameters were 1.98- $(\mathrm{P}=0.009), 3.03$ - $(\mathrm{P}=0.032)$, and 2.32-fold $(\mathrm{P}=0.018)$, respectively, indicating a 2.0 -fold is suitable for comparison with clinicopathological parameters. Accordingly, we subdivided HCCs into two groups based on TMEM165 expression employing 2.0-fold as a cut-off point. Among the 88 tumor tissues examined, $41(46.6 \%)$ exhibited $\geq 2.0$-fold increase in TMEM165 transcript expression. Consistent with the ROC analysis, higher $\alpha$-fetoprotein levels $(\geq 50 \mathrm{ng} / \mathrm{ml}$, $\mathrm{P}=0.022 ; \geq 200 \mathrm{ng} / \mathrm{ml}, \mathrm{P}=0.024)$ and presence of macroscopic vascular invasion $(\mathrm{P}=0.023)$ and serosal invasion $(\mathrm{P}=0.006)$ were significantly associated with TMEM165 overexpression ( $\geq 2.0$-fold) (Table II). In contrast, HCC-based parameters such as sex $(P=0.187)$, age $(P=0.479)$, aspatate transaminase $(\mathrm{P}=0.204)$, alanine transaminase $(\mathrm{P}=0.386)$, Child-Pugh classification $(\mathrm{P}=0.273)$, tumor size $(\mathrm{P}=0.113)$, tumor number $(\mathrm{P}=0.212)$, microscopic vascular invasion $(\mathrm{P}=0.377)$, Edmondson grade $(\mathrm{P}=0.274)$ and cirrhosis $(\mathrm{P}=0.334)$, were not associated with TMEM165 overexpression. Furthermore, HCCs classified based on significant parameters (Table II) exhibited marked differences in TMEM165 expression (Fig. 2). Specifically, the TMEM165 expression level was positively correlated with the AFP level (Fig. 2A) $(n=87, r=0.213$ and $\mathrm{P}=0.048$ ). Additionally, median increases in TMEM165 expression were 1.90- and 3.26-fold according to macroscopic vascular invasion (no and yes, $\mathrm{P}=0.034$ ) (Fig. $2 \mathrm{~B}$ ) and 1.87- and 2.95-fold according to serosal invasion (no and yes, $\mathrm{P}=0.03$ ), respectively (Fig. $2 \mathrm{C}$ ). The clinicopathological association data indicated that TMEM165-associated parameters influence aggressiveness of HCCs, based on the finding that overexpression of this protein is clinically associated with the invasive characteristics of this tumor type.

TMEM165 depletion decreases invasion of HCC cells without affecting clonal survival. In view of the clinical association of TMEM165 overexpression with macroscopic vascular and serosal invasion, we further investigated the possibility of regulatory effects on the invasion of HCC cells. Prior to evaluation, we determined the expression patterns of TMEM165 in normal and cancer cell lines to select suitable cells. For this experiment, we employed the established HCC cell lines Hep3B, Huh7, SNU354, SNU398, SNU449, SNU475 and SNU739 and hepatoblastoma cell line HepG2. Consistent with data from HCC specimens, most cancer cell lines exhibited greater expression than normal human fibroblast (BJ, Wi38, IMR90) and epithelial cells, including those of the Small Airway and Prostate (Fig. 3A).
Table II. Correlation between TMEM165 expression and clinicopathological parameters $(n=88)$.

\begin{tabular}{|c|c|c|c|}
\hline \multirow[b]{2}{*}{ Variables } & \multicolumn{2}{|c|}{ TMEM165 expression } & \multirow[b]{2}{*}{ P-value } \\
\hline & $<2.0$ fold & $\geqq 2.0$ fold & \\
\hline \multicolumn{4}{|l|}{ Sex } \\
\hline Male & 43 & 34 & 0.187 \\
\hline Female & 4 & 7 & \\
\hline \multicolumn{4}{|l|}{ Age (years) } \\
\hline$<52$ & 20 & 19 & 0.479 \\
\hline$\geq 52$ & 26 & 22 & \\
\hline $\mathrm{AFP}(\mathrm{ng} / \mathrm{ml})$ & & & 0.149 \\
\hline$<20$ & 23 & 15 & \\
\hline$\geq 20$ & 23 & 26 & \\
\hline \multicolumn{4}{|l|}{ AFP (ng/ml) } \\
\hline$<50$ & 30 & 17 & 0.022 \\
\hline$\geq 50$ & 16 & 24 & \\
\hline AFP (ng/ml) & & & 0.024 \\
\hline$<200$ & 34 & 21 & \\
\hline$\geq 200$ & 12 & 20 & \\
\hline \multicolumn{4}{|l|}{ AST (U/l) } \\
\hline$<40$ & 20 & 22 & 0.204 \\
\hline$\geq 40$ & 27 & 19 & \\
\hline \multicolumn{4}{|l|}{ ALT (U/l) } \\
\hline$<35$ & 17 & 17 & 0.386 \\
\hline$\geq 35$ & 30 & 24 & \\
\hline \multicolumn{4}{|c|}{ Child-Pugh classification } \\
\hline A & 36 & 34 & 0.273 \\
\hline $\mathrm{B}, \mathrm{C}$ & 5 & 2 & \\
\hline \multicolumn{4}{|c|}{ Tumor size $(\mathrm{cm})$} \\
\hline$<5$ & 26 & 16 & 0.113 \\
\hline$\geq 5$ & 21 & 24 & \\
\hline \multicolumn{4}{|c|}{ Tumor number } \\
\hline Single & 33 & 26 & 0.212 \\
\hline Multiple & 7 & 10 & \\
\hline \multicolumn{4}{|c|}{$\begin{array}{l}\text { Macroscopic vascular } \\
\text { invasion }\end{array}$} \\
\hline No & 43 & 35 & 0.023 \\
\hline Yes & 0 & 5 & \\
\hline \multicolumn{4}{|c|}{$\begin{array}{l}\text { Microscopic vascular } \\
\text { invasion }\end{array}$} \\
\hline No & 17 & 17 & 0.377 \\
\hline Yes & 22 & 17 & \\
\hline \multicolumn{4}{|c|}{ Serosal invasion } \\
\hline No & 41 & 27 & 0.006 \\
\hline Yes & 2 & 10 & \\
\hline \multicolumn{4}{|c|}{ Edmondson grade } \\
\hline $\mathrm{I}, \mathrm{II}$ & 33 & 26 & 0.274 \\
\hline III,IV & 13 & 15 & \\
\hline \multicolumn{4}{|l|}{ Cirrhosis } \\
\hline No & 28 & 22 & 0.334 \\
\hline Yes & 14 & 15 & \\
\hline
\end{tabular}

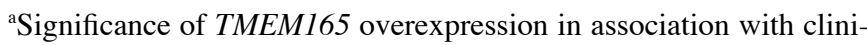
copathological parameters was calculated using the Chi-Square test; ${ }^{b}$ Bold indicates $\mathrm{P}<0.05$. AFP, $\alpha$-fetoprotein; AST, aspartate aminotransferase; ALT, alanine transaminase. 
A

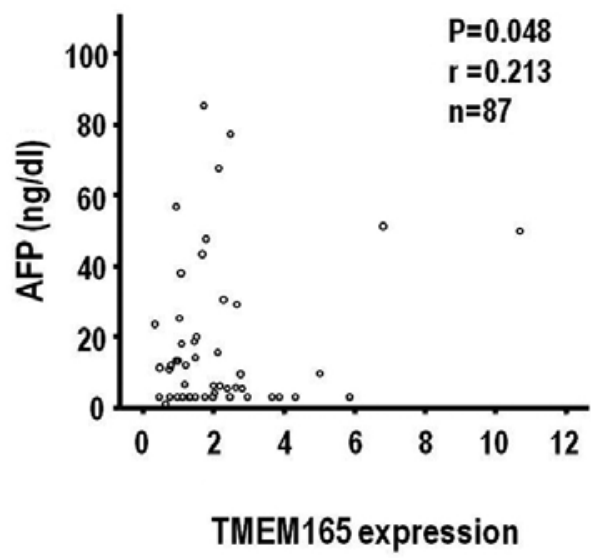

B
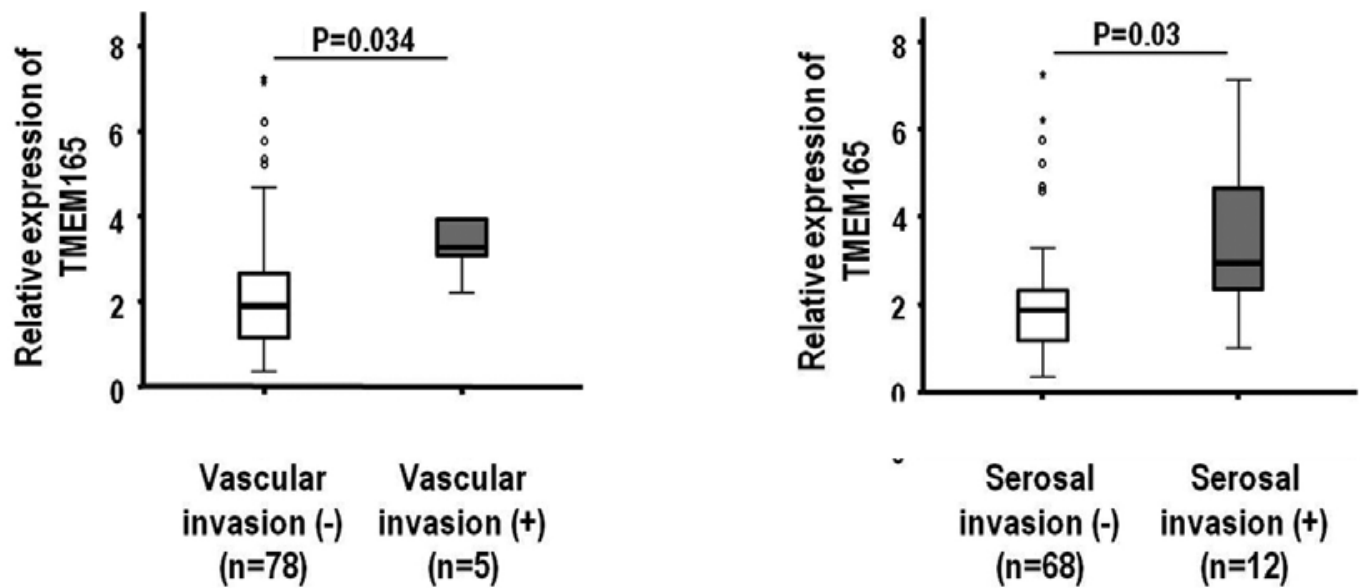

Figure 2. TMEM165 expression in HCC is correlated with aggressive clinical parameters. (A) The correlation between the levels of TMEM165 and AFP was analyzed using Pearson's correlation test. (B and C) HCC samples were classified into two subgroups based on significant clinicopathological parameters (Table II), including (B) macroscopic vascular invasion and (C) serosal invasion. Using real-time RT-PCR results from Fig. 1B, differences in TMEM165 mRNA levels between the subgroups were analyzed by a two-sample t-test. Open circle $\left(^{\circ}\right)$ and asterisk (*) are the outlier and extreme outlier samples, respectively, with $95 \%$ confidence interval and the horizontal line indicates median value. TMEM165, transmembrane protein 165; HCC, human hepatocellular carcinoma; AFP, $\alpha$-fetoprotein.

Next, we examined the effect of TMEM165 depletion on invasion of Huh7 cells that express high levels of TMEM165. Notably, TMEM165 depletion achieved via transfection of siRNA induced a significant decrease in the invasive activity of Huh7 cells (Fig. 3B). Two siRNAs (\#1 and \#2) recognizing different regions on the coding sequence induced a noticeable level of suppression of invasive activity (51.3 and $72.1 \%$, respectively, compared to the control siRNA). Significantly reduced invasion was additionally observed in the SNU475 cell line (Fig. 3C). The rates of inhibition of invasion by TMEM165 siRNA \#1 and \#2 were 88.2 and $95.7 \%$, respectively, clearly indicating that knockdown of TMEM165 in HCC suppresses invasive activity to a significant extent. To further confirm this finding, we assessed the effects of TMEM165 depletion on clonal growth that can detect cell proliferation and death. Notably, clonal growth was hardly inhibited in both cell lines after transfection of TMEM165 siRNAs (P>0.1, Fig. 4A and B). Thus, the TMEM165 depletion-mediated suppression of HCC cell invasion activity was not attributable to decreased clonal survival.

TMEM165 affects matrix metalloproteinase-2 (MMP-2) expression. To further determine the mechanisms underlying TMEM165-mediated regulation of invasion, we examined the potential involvement of MMPs, well-known invasion activators. Specifically, we assessed the expression levels of $M M P-1,-2$ and -9 in HCC cells depleted of TMEM165. As expected from the cell invasion data, $M M P-2$ levels were severely decreased under conditions of TMEM165 depletion in both Huh7 and SNU475 cell lines (Fig. 5A and B). In contrast, $M M P-1$ and -9 levels were not affected. To determine whether TMEM165 controls cancer cell invasion in a similar manner to Golgi phosphoprotein 2, we examined the expression of $M M P-13$ that are regulated by Golgi phosphoprotein 2 (21). Notably, TMEM165 depletion had no effect on the expression patterns of this molecule, indicating that the mechanism by 
A

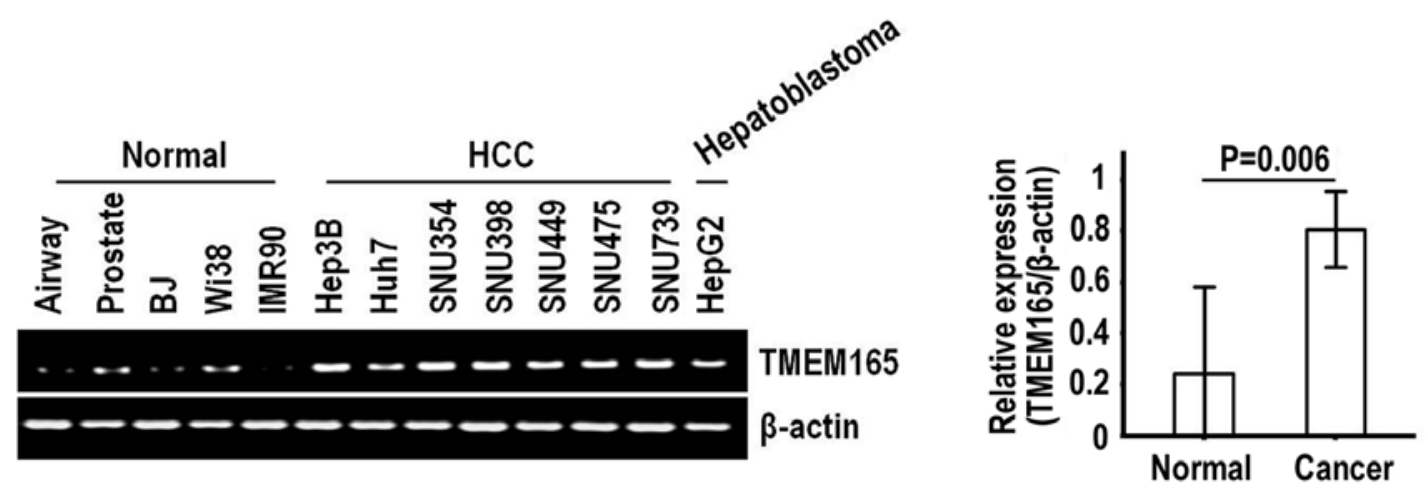

B

\section{Huh7}
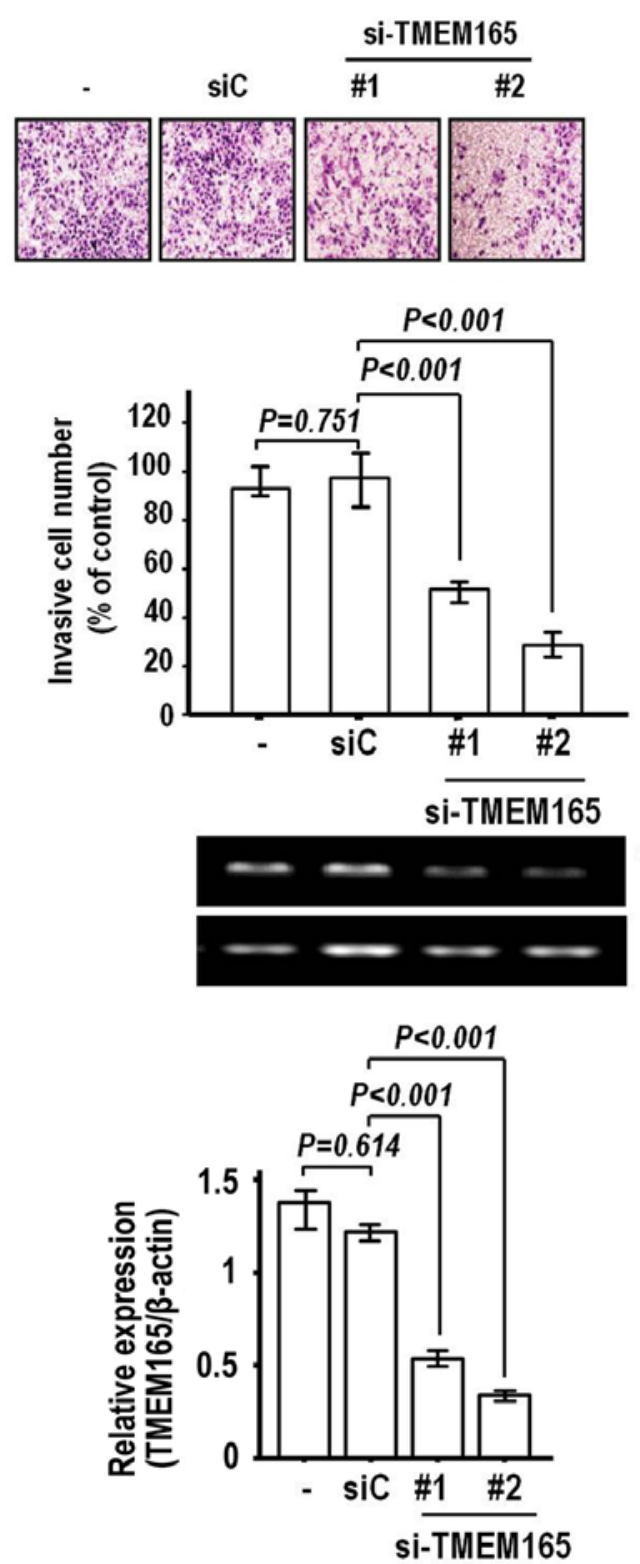

C

\section{SNU475}
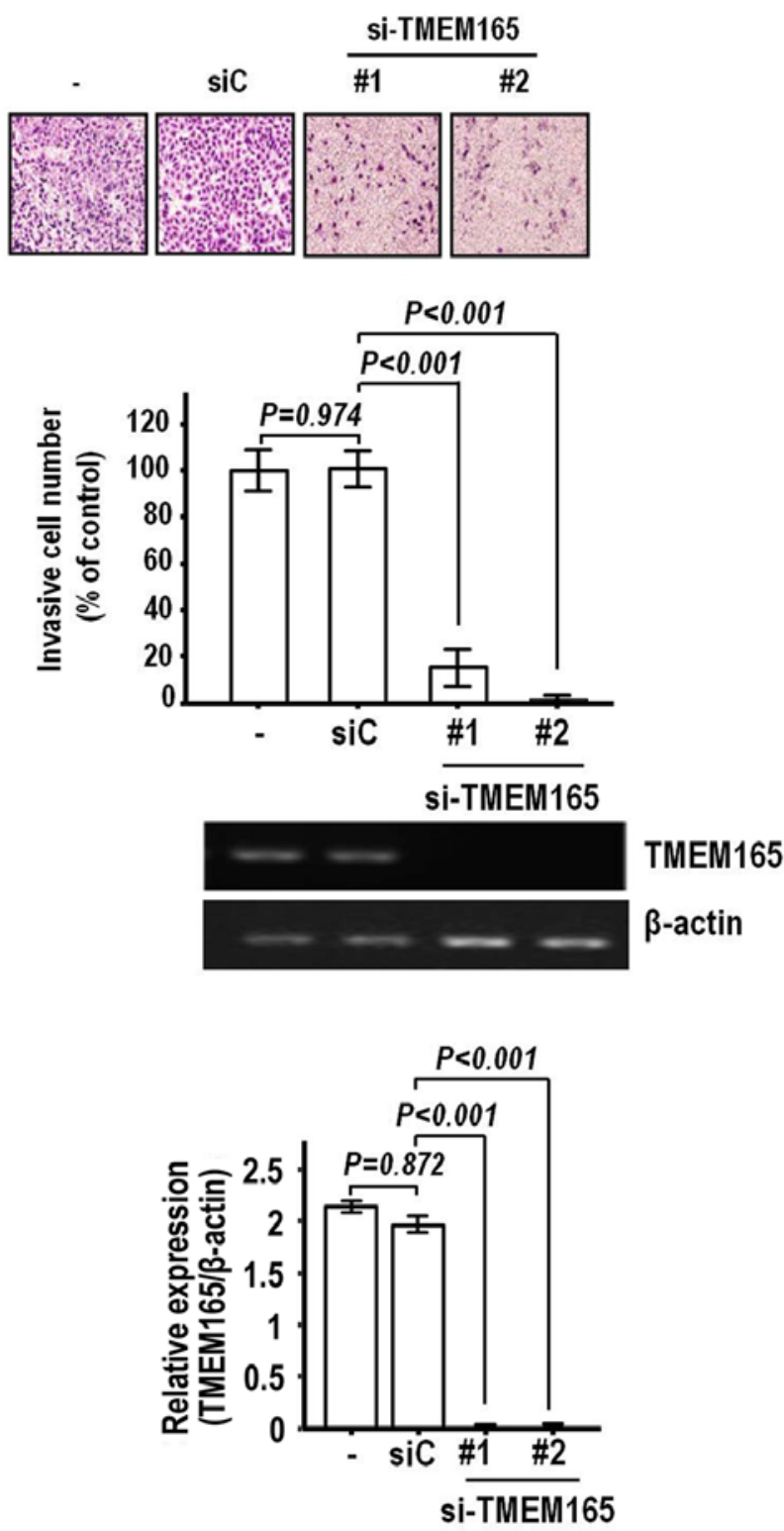

Figure 3. TMEM165 depletion weakens the invasive activity of HCC cells. (A) TMEM165 expression was assessed in normal human fibroblasts, epithelial cells and various HCC cell lines via semi-quantitative RT-PCR using $\beta$-actin as an internal control. (B and C) TMEM165 siRNA (\#1 and \#2) or control-siRNA (siC)-transfected Huh7 (B) and SNU475 cells (C) were loaded onto a Matrigel-coated invasion chamber. Invading cells were stained with crystal violet and visualized under a microscope (magnification, x100). RT-PCR analysis of TMEM165 depletion following siRNA transfection. Invasion rates were calculated by dividing the counts (invading cells) of TMEM165-transfected cells with those of control siRNA-transfected cells. The mean \pm SD of calculated cells were plotted, with P-values calculated by ANOVA with Dunnett's test. The densitometry analysis was performed using ImageJ software. TMEM165, transmembrane protein 165; HCC, human hepatocellular carcinoma. 
A Huh7
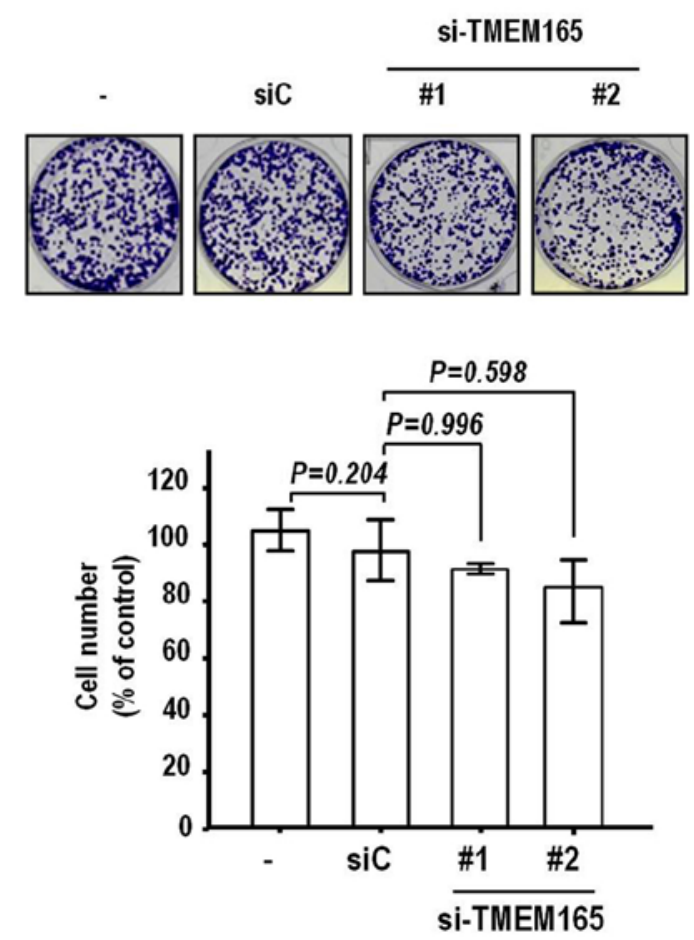

B
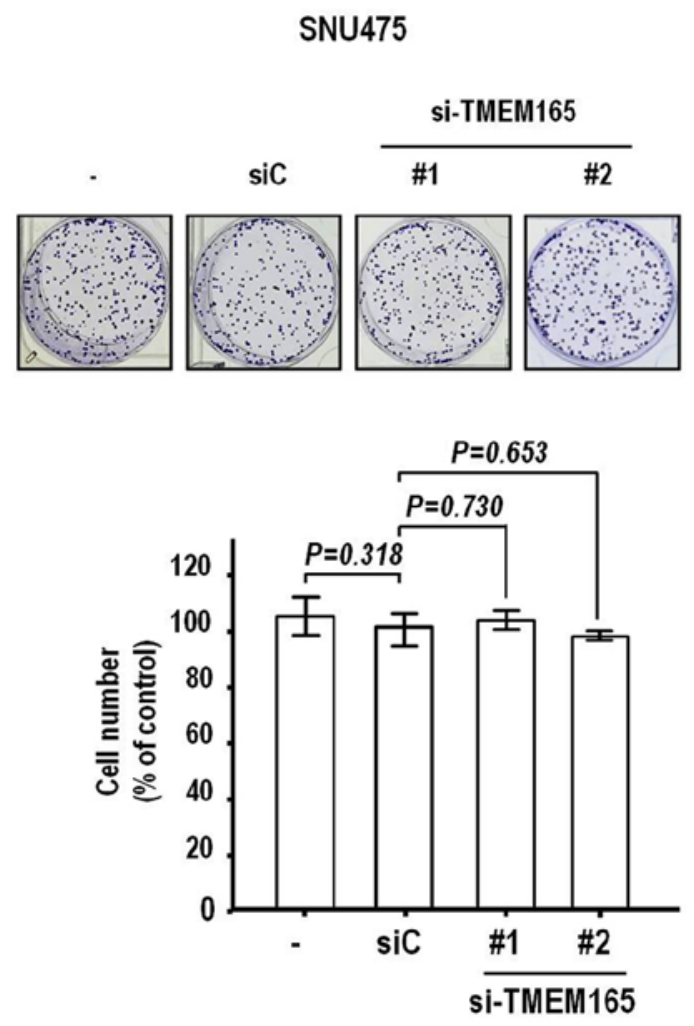

Figure 4. TMEM165 depletion has no impact on clonal survival of HCC cells. siRNA-transfected Huh7 (A) and SNU475 cells (B) were seeded at low density, and the surviving colonies were stained with crystal violet and visualized under a microscope (magnification, x100). Survival rates were calculated by dividing the counts (surviving cells) of TMEM165-siRNA (\#1 and \#2)-transfected cells with those of control siRNA (siC)-transfected cells. Bars represent the mean $\pm \mathrm{SD}$, with P-values calculated by ANOVA with Dunnett's test. TMEM165, transmembrane protein 165; HCC, human hepatocellular carcinoma.
A

Huh7

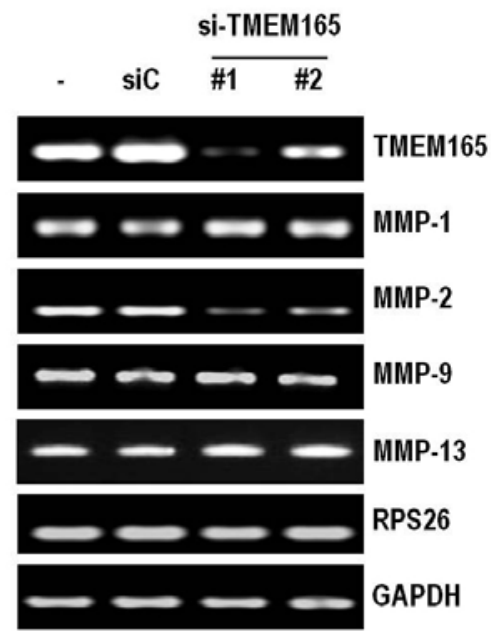

B

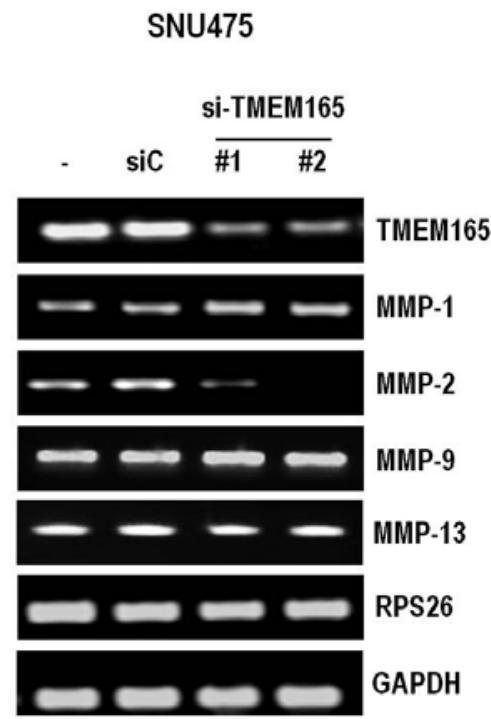

Figure 5. TMEM165 controls MMP-2 expression. TMEM165-depleted Huh7 (A) and SNU475 cells (B) were prepared by transfection with targeted siRNAs and grown in 5\% serum for $48 \mathrm{~h}$. MMP-1, $-2,-9$ and -13 mRNA levels were assessed using RT-PCR. Ribosomal protein S26 (RPS26) and GAPDH were used as internal controls. TMEM165, transmembrane protein 165; MMP, matrix metalloproteinase.

which TMEM165 promotes the invasive activity of cancer cells is distinct from that of Golgi phosphoprotein 2.

\section{Discussion}

The TMEM165 protein, mainly characterized in association with congenital disorders of glycosylation type 2 disease, has recently attracted significant research attention $(13,14,22,23)$. Based on cohort studies, 3 different mutations in TMEM165 have been reported to date, specifically, homozygous point mutation in the deep intronic splice region, homozygous missense mutation and heterozygous missense mutation, all of which culminate in loss or decrease in function of the protein $(11,14)$. Recent in vitro studies have disclosed that TMEM165 is associated with Golgi homeostasis sensitive to 
manganese concentrations (18), indicating that mutation and loss of function of the protein can trigger defects in manganese-sensitive Golgi homeostasis. The importance of Golgi proteins in promoting cancer progression has been highlighted in previous studies. Golgi phosphoprotein 2 (GP73) is aberrantly expressed in HCC and knockdown of this gene inhibits cancer cell invasiveness via altering expression of E-cadherin, $\mathrm{N}$-cadherin and MMP-13 $(24,25)$. Moreover, Golgi phosphoprotein 3 (GOLPH3) is overexpressed in various cancer types, in turn, increasing invasion and migration of cancer cells (26-28). GOLPH3 interacts with phosphatidylinositol-4-phosphate and myosin18A to facilitate Golgi to plasma membrane trafficking and regulates the directional migration of cells (29). Despite the recent focus on Golgi proteins, the specific function of TMEM165 in cancer remains to be determined.

We previously established an integrative analysis tool to determine the gene expression patterns between non-tumor and tumor regions in correlation with clinical outcomes (6). Based on these experiments, we demonstrated for the first time that the TMEM165 protein is significantly upregulated in tumor compared to non-tumor tissues of HCC patients. Notably, overexpression of TMEM165 (using the 2-fold cut-off criterion) was positively correlated with aggressiveness of $\mathrm{HCC}$ characterized by high AFP levels as well as presence of macroscopic vascular and microscopic serosal invasion. In keeping with this finding, depletion of TMEM165 significantly reduced invasiveness of human HCC cell lines in vitro, accompanied by a decrease in expression of $M M P-2$, an endopeptidase that degrades the extracellular matrix to stimulate cancer invasion and metastasis. However, other MMPs (MMP-1, -9, and -13) were not affected by depletion of TMEM165. These results support a role of TMEM165 as a key molecule that directs cancer cell invasion, consequently promoting aggressiveness. Clonal survival analysis can be effectively used to detect several phenotypic changes, including proliferation, death and senescence. TMEM165 depletion decreased cancer cell invasion without affecting clonal survival, indicative of a negligible effect on these phenotypes. Inhibition of cellular proliferation, death or senescence is reported to trigger a decrease in the invasion rate of cancer cells (30). Therefore, TMEM165 regulation of cancer cell invasion is credible, since the possibility that phenotypic changes, such as cellular proliferation, death, and senescence, can affect cancer cell invasion was eliminated.

Despite the significant correlation between TMEM165 overexpression and aggressiveness of the tumor phenotype, TMEM165 was not associated with patient prognosis in the current study (data not shown). This may be attributable to the small number of samples used for our analysis. Studies on larger cohorts are therefore required to validate the expression patterns of TMEM165 in relation to patient prognosis. Furthermore, while decreased invasiveness of cancer cells upon TMEM165 knockdown was evident, the precise mechanisms require elucidation in future studies. Considering the relationship between TMEM165 mutation and type II congenital disorder of glycosylation, it is plausible that its overexpression in HCC is associated with abnormalities in galactosylation and sialylation of $\mathrm{N}$-glycoproteins observed in the disease. These protein modifications influence the invasive activity of cancer cells (31). Several invasion-related proteins (CD44, integrin and E-cadherin) have been revealed to be markedly affected by glycosylation (32-34). In summary, TMEM165 is both transcriptionally and translationally overexpressed in HCC and associated with invasive activity.

\section{Acknowledgements}

Not applicable.

\section{Funding}

The present study was supported by grants from the National Research Foundation of Korea (nos. 2012M3A9B6055346, 2017M3A9A8033561 and 2017R1A2B4008805) and the Korea Institute of Radiological and Medical Sciences (nos. 50531-2018 and 50542-2018) funded by the Ministry of Science, ICT and Future Planning, Republic of Korea.

\section{Availability of data and materials}

The datasets used during the present study are available from the corresponding author upon reasonable request.

\section{Authors' contributions}

KHL, SBK and JH conceived and supervised the study. KHL, JSL and MYK wrote the manuscript. SBK and ERP reviewed and edited manuscript. MYK, JSL, ERP, YNS and JYJ prepared material and/or performed experiment. JSL and MYK performed statistical analysis. EHC, SHP, CJH, DWC, JJJ, KSS and SBK generated clinical data. All authors read and approved the manuscript and agree to be accountable for all aspects of the research in ensuring that the accuracy or integrity of any part of the work are appropriately investigated and resolved.

\section{Ethics approval and consent to participate}

The present study was approved by the Institutional Review Boards of Korea Cancer Center Hospital and Seoul National University Hospital. Written informed consent was either waived by the Institutional Review Board of Korea Cancer Center Hospital or received from patients at Seoul National University Hospital, respectively.

\section{Patient consent for publication}

Not applicable.

\section{Competing interests}

The authors declare that they have no competing interests.

\section{References}

1. Torre LA, Bray F, Siegel RL, Ferlay J, Lortet-Tieulent J and Jemal A: Global cancer statistics, 2012. CA Cancer J Clin 65: $87-108,2015$

2. El-Serag HB: Hepatocellular carcinoma. N Engl J Med 365: 1118-1127, 2011.

3. Bruix J and Sherman M: American Association for the Study of Liver Diseases: Management of hepatocellular carcinoma: An update. Hepatology 53: 1020-1022, 2011. 
4. Llovet JM, Ricci S, Mazzaferro V, Hilgard P, Gane E, Blanc JF, de Oliveira AC, Santoro A, Raoul JL, Forner A, et al: Sorafenib in advanced hepatocellular carcinoma. N Engl J Med 359: 378-390, 2008

5. Cheng AL, Kang YK, Chen Z, Tsao CJ, Qin S, Kim JS, Luo R, Feng J, Ye S, Yang TS, et al: Efficacy and safety of sorafenib in patients in the Asia-Pacific region with advanced hepatocellular carcinoma: A phase III randomised, double-blind, placebo-controlled trial. Lancet Oncol 10: 25-34, 2009.

6. Kim BY, Suh KS, Lee JG, Woo SR, Park IC, Park SH, Han CJ, Kim SB, Jeong SH, Yeom YI, et al: Integrated analysis of prognostic gene expression profiles from hepatitis B virus-positive hepatocellular carcinoma and adjacent liver tissue. Ann Surg Oncol 19 (Suppl 3): S328-S338, 2012.

7. Kim BY, Choi DW, Woo SR, Park ER, Lee JG, Kim SH, Koo I, Park SH, Han CJ, Kim SB, et al: Recurrence-associated pathways in hepatitis B virus-positive hepatocellular carcinoma BMC Genomics 16: 279, 2015.

8. Park ER, Kim SB, Lee JS, Kim YH, Lee DH, Cho EH, Park SH, Han CJ, Kim BY, Choi DW, et al: The mitochondrial hinge protein, UQCRH, is a novel prognostic factor for hepatocellular carcinoma. Cancer Med 6: 749-760, 2017.

9. Potelle S, Morelle W, Dulary E, Duvet S, Vicogne D, Spriet C, Krzewinski-Recchi MA, Morsomme P, Jaeken J, Matthijs G, et al: Glycosylation abnormalities in Gdt1p/TMEM165 deficient cells result from a defect in Golgi manganese homeostasis. Hum Mol Genet 25: 1489-1500, 2016.

10. Rosnoblet C, Legrand D, Demaegd D, Hacine-Gherbi H, de Bettignies G, Bammens R, Borrego C, Duvet S, Morsomme P, Matthijs G, et al: Impact of disease-causing mutations on TMEM165 subcellular localization, a recently identified protein involved in CDG-II. Hum Mol Genet 22: 2914-2928, 2013.

11. Foulquier F, Amyere M, Jaeken J, Zeevaert R, Schollen E, Race V, Bammens R, Morelle W, Rosnoblet C, Legrand D, et al TMEM165 deficiency causes a congenital disorder of glycosylation. Am J Hum Genet 91: 15-26, 2012

12. Bammens R, Mehta N, Race V, Foulquier F, Jaeken J, Tiemeyer M, Steet R, Matthijs G and Flanagan-Steet $H$ : Abnormal cartilage development and altered N-glycosylation in Tmem165-deficient zebrafish mirrors the phenotypes associated with TMEM165-CDG. Glycobiology 25: 669-682, 2015.

13. Schulte Althoff S, Grüneberg M, Reunert J, Park JH, Rust S, Mühlhausen C, Wada Y, Santer R and Marquardt T: TMEM165 deficiency: Postnatal changes in Glycosylation. JIMD Rep 26: 21-29, 2016.

14. Dulary E, Potelle S, Legrand D and Foulquier F: TMEM165 deficiencies in Congenital Disorders of Glycosylation type II (CDG-II): Clues and evidences for roles of the protein in Golg functions and ion homeostasis. Tissue Cell 49: 150-156, 2017.

15. Jaeken J, Schachter H, Carchon H, De Cock P, Coddeville B and Spik G: Carbohydrate deficient glycoprotein syndrome type II: A deficiency in Golgi localised N-acetyl-glucosaminyltransferase II. Arch Dis Child 71: 123-127, 1994.

16. Peters V, Penzien JM, Reiter G, Körner C, Hackler R, Assmann B, Fang J, Schaefer JR, Hoffmann GF and Heidemann PH: Congenital disorder of glycosylation IId (CDG-IId) - A new entity: Clinical presentation with Dandy-Walker malformation and myopathy. Neuropediatrics 33: 27-32,2002.

17. Martinez-Duncker I, Dupré T, Piller V, Piller F, Candelier JJ, Trichet C, Tchernia G, Oriol R and Mollicone R: Genetic complementation reveals a novel human congenital disorder of glycosylation of type II, due to inactivation of the Golgi CMP-sialic acid transporter. Blood 105: 2671-2676, 2005

18. Potelle S, Dulary E, Climer L, Duvet S, Morelle W, Vicogne D, Lebredonchel E, Houdou M, Spriet C, Krzewinski-Recchi MA, et al: Manganese-induced turnover of TMEM165. Biochem J 474: 1481-1493, 2017.
19. Marrero JA, Romano PR, Nikolaeva O, Steel L, Mehta A, Fimmel CJ, Comunale MA, D'Amelio A, Lok AS and Block TM: GP73, a resident Golgi glycoprotein, is a novel serum marker for hepatocellular carcinoma. J Hepatol 43: 1007-1012, 2005.

20. Wei H, Li B, Zhang R, Hao X, Huang Y, Qiao Y, Hou J, Li X and Li X: Serum GP73, a marker for evaluating progression in patients with chronic HBV infections. PLoS One 8: e53862, 2013.

21. Jin D, Tao J, Li D, Wang Y, Li L, Hu Z, Zhou Z, Chang X, Qu C and Zhang H: Golgi protein 73 activation of MMP-13 promotes hepatocellular carcinoma cell invasion. Oncotarget 6: 33523-33533, 2015.

22. Krzewinski-Recchi MA, Potelle S, Mir AM, Vicogne D, Dulary E, Duvet S, Morelle W, de Bettignies G and Foulquier F: Evidence for splice transcript variants of TMEM165, a gene involved in CDG. Biochim Biophys Acta 1861: 737-748, 2017.

23. Morelle W, Potelle S, Witters P, Wong S, Climer L, Lupashin V, Matthijs G, Gadomski T, Jaeken J, Cassiman D, et al: Galactose supplementation in patients with TMEM165-CDG rescues the glycosylation defects. J Clin Endocrinol Metab 102: 1375-1386, 2017.

24. Chen X, Wang Y, Tao J, Shi Y, Gai X, Huang F, Ma Q, Zhou Z, Chen H, Zhang H, et al: mTORC1 up-regulates GP73 to promote proliferation and migration of hepatocellular carcinoma cells and growth of xenograft tumors in mice. Gastroenterology 149: 741-752, 2015.

25. Yang Y, Liu Q, Zhang H, Zhao H, Mao R, Li Z, Ya S, Jia C and Bao Y: Silencing of GP73 inhibits invasion and metastasis via suppression of epithelial-mesenchymal transition in hepatocellular carcinoma. Oncol Rep 37: 1182-1188, 2017.

26. Ma Y, Wang X, Wu Y, Sun B, Lv H, Rong F and Zheng X: Overexpression of GOLPH3 protein is associated with worse prognosis in patients with epithelial ovarian cancer. Tumour Biol 35: 11845-11849, 2014

27. Li Q, Ma Y and Xu W: High GOLPH3 expression is associated with poor prognosis and invasion of hepatocellular carcinoma. Mol Med Rep 11: 4315-4320, 2015.

28. Li W, Guo F, Gu M, Wang G, He X, Zhou J, Peng Y, Wang Z and Wang X: Increased expression of GOLPH3 is associated with the proliferation of prostate cancer. J Cancer 6: 420-429, 2015.

29. Taft MH, Behrmann E, Munske-Weidemann LC, Thiel C, Raunser S and Manstein DJ: Functional characterization of human myosin-18A and its interaction with F-actin and GOLPH3. J Biol Chem 288: 30029-30041, 2013.

30. Schaeffer D, Somarelli JA, Hanna G, Palmer GM and Garcia-Blanco MA: Cellular migration and invasion uncoupled: Increased migration is not an inexorable consequence of epithelial-to-mesenchymal transition. Mol Cell Biol 34: 3486-3499, 2014

31. Pinho SS and Reis CA: Glycosylation in cancer: Mechanisms and clinical implications. Nat Rev Cancer 15: 540-555, 2015.

32. English NM, Lesley JF and Hyman R: Site-specific de-N-glycosylation of CD44 can activate hyaluronan binding, and CD44 activation states show distinct threshold densities for hyaluronan binding. Cancer Res 58: 3736-3742, 1998.

33. Guo HB, Lee I, Kamar M, Akiyama SK and Pierce M: Aberrant $\mathrm{N}$-glycosylation of beta1 integrin causes reduced alpha5beta1 integrin clustering and stimulates cell migration. Cancer Res 62: 6837-6845, 2002

34. Pinho SS, Seruca R, Gärtner F, Yamaguchi Y, Gu J, Taniguchi N and Reis CA: Modulation of E-cadherin function and dysfunction by N-glycosylation. Cell Mol Life Sci 68: 1011-1020, 2011.

This work is licensed under a Creative Commons Attribution-NonCommercial-NoDerivatives 4.0 International (CC BY-NC-ND 4.0) License. 